\title{
Theory and Applications of the Fission Matrix Method for Continuous-Energy Monte Carlo
}

\author{
Sean Carney ${ }^{\mathrm{a}, *}$, Forrest Brown ${ }^{\mathrm{b}}$, Brian Kiedrowski ${ }^{\mathrm{b}}$, William Martin ${ }^{\mathrm{a}}$ \\ ${ }^{a}$ University of Michigan, 2355 Bonisteel Boulevard, Ann Arbor, MI 48109, USA \\ ${ }^{b}$ Los Alamos National Laboratory, P.O. Box 1663 MS A143, Los Alamos, NM 87545, USA
}

\begin{abstract}
The fission matrix method can be used to provide estimates of the fundamental mode fission distribution, the dominance ratio, the eigenvalue spectrum, and higher mode forward and adjoint eigenfunctions of the fission distribution. It can also be used to accelerate the convergence of power method iterations and to provide basis functions for higherorder perturbation theory. The higher-mode fission sources can be used to determine higher-mode forward fluxes and tallies, and work is underway to provide higher-mode adjoint-weighted fluxes and tallies. These aspects of the method are here both theoretically justified and demonstrated, and then used to investigate fundamental properties of the transport equation for a continuous-energy physics treatment. Implementation into the MCNP6 Monte Carlo code is also discussed, including a sparse representation of the fission matrix, which permits much larger and more accurate representations. Properties of the calculated eigenvalue spectrum of a 2D PWR problem are discussed: for a fine enough mesh and a sufficient degree of sampling, the spectrum both converges and has a negligible imaginary component. Calculation of the fundamental mode of the fission matrix for a fuel storage vault problem shows how convergence can be accelerated by over a factor of ten given a flat initial distribution. Forward fluxes and the relative uncertainties for a 2D PWR are shown, both of which qualitatively agree with expectation. Lastly, eigenmode expansions are performed during source convergence of the 2D PWR problem for two initial distributions; observed decay rates of coefficients agree closely with expectation.
\end{abstract}

Keywords: Monte Carlo, criticality, k-effective, eigenmodes

\section{Introduction}

Continuous-energy Monte Carlo codes simulate neutron behavior using the best available nuclear data, accurate physics models, and detailed geometry models. Reactor criticality calculations for $k_{e f f}$ and the power distribution are carried out iteratively, using the power method, where batches of neutrons are simulated for a single generation. The first-generation fission neutrons produced in a batch become the starting neutron sites for the next batch. A suitable number of "inactive" initial batches are required to converge to the fundamental mode eigenvalue and eigenfunction, and then succeeding iterations with "active" batches are used to accumulate Monte Carlo tallies for estimating desired reaction rate distributions.

${ }^{*}$ Corresponding author. Present address: University of Michigan, 2355 Bonisteel Boulevard, Ann Arbor, MI, 48109, USA. Tel.: +1 (248) 7099132

Email address: seanec@umich.edu (Sean Carney)
Most Monte Carlo codes perform the power iteration without acceleration and can sometimes exhibit very slow convergence. Statistical noise for batch results precludes the use of common outer iteration acceleration methods (e.g. Chebyshev). An additional limitiation of standard Monte Carlo codes is the inability to directly calculate higher eigenmodes.

The fission matrix approach was proposed in the earliest works on Monte Carlo criticality calculations (Morton (1956); Kaplan (1958); Hammersely and Handscomb (1964)) and has been tried by many researchers over the years (Urbatsch (1992); Kitada and Takeda (2001); Dufek and Gudowski (2009); Wenner and Haghighat (2011)). The present work provides a rigorous derivation of the forward and adjoint forms of the fission matrix treatment for $K$-eigenvalue problems. The method is then used to investigate fundamental properties of the transport equation for a continuousenergy physics treatment, for both forward and adjoint modes. The eigenvalue spectrum of a 2D PWR problem 
is examined in terms of its convergence with mesh refinement and the diminishment of its complex part with larger sampling.

Implementation in the MCNP6 Monte Carlo code (Goorley et. al. (2013)) and the sparse storage methodology is discussed, as well as the ability to compute higher mode eigenfunctions for fission sources, fluxes, and reaction rates (e.g. a higher mode capture rate for use in second-order perturbation theory calculations). Results from these higher mode calculations are shown, in addition to two applications relevant to criticality calculations: source convergence acceleration and modal expansion. Using forward source modes from the fission matrix, the first 30 forward flux modes are calculated from a 2D PWR problem by running fixed source calculations, and reasonable relative variances are found. Convergence acceleration is demonstrated for a fuel storage vault problem: from a flat initial distribution, the number of iterations required for convergence is reduced by over a factor of ten. Eigenmode expansions of converging fission sources for the 2D PWR problem are discussed for two different initial distributions: a point in the center of the core and a point in the corner. The decay rates of significant expansion coefficients are shown to agree with expectations calculated directly from the fission matrix. This paper will summarize and extend work reported in Carney et. al. (2012); Brown et. al. (2013); Carney et. al. (2013a,b).

\section{Theory}

The following sections provide derivations of the $K$ effective form of the integral transport equations for the forward and adjoint fission sources, for continuousenergy problems using a rigorous Green's function approach (Sections II.A-II.C). The integral equations are then integrated over spatial regions to provide an exact prescription for the fission matrix elements and resulting equations for the regionwise sources (Sections II.D-II.E). The solution to the fission matrix equations, both forward and adjoint, is shown to be exact if the within-region weighting functions are known (as in the case of a converged fission source distribution), or in the limit of vanishingly small region size (Section II.F). The basis for higher eigenmode calculation is then mentioned (Section II.G), followed by a discussion of the method's implementation into MCNP6 (Sections II.H-II.K). Lastly, the expansion of the iterative fission source into fission source eigenmodes is introduced (Section II.I).

\section{II.A. Integral Equation for Neutron Source}

The $K$-eigenvalue form of the neutron transport equation is

$$
\mathbf{M} \cdot \Psi(\vec{r}, E, \hat{\Omega})=\frac{1}{K} \frac{\chi(E)}{4 \pi} S(\vec{r}),
$$

where $\mathbf{M}$ is the net loss operator defined by

$$
\begin{gathered}
\mathbf{M} \cdot \Psi(\vec{r}, E, \hat{\Omega})=\hat{\Omega} \cdot \nabla \Psi(\vec{r}, E, \hat{\Omega})+\Sigma_{T}(\vec{r}, E) \Psi(\vec{r}, E, \hat{\Omega}) \\
-\iint d E^{\prime} d \hat{\Omega}^{\prime} \Sigma_{S}\left(\vec{r}, E^{\prime} \rightarrow E, \hat{\Omega}^{\prime} \rightarrow \hat{\Omega}\right) \Psi\left(\vec{r}, E^{\prime}, \hat{\Omega}^{\prime}\right),
\end{gathered}
$$

which contains leakage, collision, and scattering terms, respectively. $S(\vec{r})$ is the fission neutron source, defined by

$$
S(\vec{r})=\iint d E^{\prime} d \hat{\Omega}^{\prime} v \Sigma_{F}\left(\vec{r}, E^{\prime}\right) \Psi\left(\vec{r}, E^{\prime}, \hat{\Omega}^{\prime}\right),
$$

and $\chi(E)$ is the emission energy spectrum of fission neutrons. Fission neutron emission is assumed to be isotropic. To simplify the analysis that follows, $\chi(E)$ is assumed to be independent of space and the energy of the neutrons causing fission. (See Appendix A for discussion.)

The Green's function for this problem is defined by the equation

$$
\begin{aligned}
\mathbf{M} \cdot G\left(\vec{r}_{0}, E_{0}, \hat{\Omega}_{0} \rightarrow\right. & \vec{r}, E, \hat{\Omega})= \\
& \delta\left(\vec{r}-\vec{r}_{0}\right) \delta\left(E-E_{0}\right) \delta\left(\hat{\Omega}-\hat{\Omega}_{0}\right),
\end{aligned}
$$

where the " 0 " subscript denotes an initial point in phase space, and $\delta$ is the Dirac delta function. Then, based on linearity of the transport equation and the superposition principle, it follows that

$$
\begin{aligned}
\Psi(\vec{r}, E, \hat{\Omega})=\frac{1}{K} \iiint d \vec{r}_{0} d E_{0} d \hat{\Omega}_{0} \frac{\chi\left(E_{0}\right)}{4 \pi} \\
\cdot S\left(\vec{r}_{0}\right) G\left(\vec{r}_{0}, E_{0}, \hat{\Omega}_{0} \rightarrow \vec{r}, E, \hat{\Omega}\right) .
\end{aligned}
$$

Eq. (5) is the $K$-eigenvalue form of the Peierls equation. Now multiply Eq. (5) by $v \Sigma_{F}(\vec{r}, E)$ and integrate over all $E$ and $\hat{\Omega}$ :

$$
S(\vec{r})=\frac{1}{K} \int d \vec{r}_{0} S\left(\vec{r}_{0}\right) H\left(\vec{r}_{0} \rightarrow \vec{r}\right),
$$

where the kernel $H\left(\vec{r}_{0} \rightarrow \vec{r}\right)$ represents the energy-angle averaged Green's function,

$$
\begin{array}{r}
H\left(\vec{r}_{0} \rightarrow \vec{r}\right)=\iiint \int_{\int} d E d \hat{\Omega} d E_{0} d \hat{\Omega}_{0} v \Sigma_{F}(\vec{r}, E) \\
\cdot \frac{\chi\left(E_{0}\right)}{4 \pi} G\left(\vec{r}_{0}, E_{0}, \hat{\Omega}_{0} \rightarrow \vec{r}, E, \hat{\Omega}\right) .
\end{array}
$$


Eq. (6) is an integral equation for the neutron source at $\vec{r}$ expressed in terms of the kernel $H$. $H$ is the Green's function integrated over angles and energies, weighted by the initial spectrum and final fission neutron production. It can readily be evaluated by either continuousenergy or multigroup Monte Carlo without approximation. That is, the Green's function $G$ is provided directly by the transport simulation in a Monte Carlo code; the energy-angle integration to produce $H$ in Eq. (7) is a tally in the Monte Carlo simulation, binned according to the initial and final spatial positions. No approximations were made in obtaining Eqs. (6) and (7).

\section{II.B. Integral Equation for Adjoint Neutron Source}

The $K$-eigenvalue form of the adjoint neutron transport equation can be written as

$$
\mathbf{M}^{\dagger} \cdot \Psi^{\dagger}(\vec{r}, E, \hat{\Omega})=\frac{1}{K} v \Sigma_{F}(\vec{r}, E) S^{\dagger}(\vec{r}),
$$

where $\mathbf{M}^{\dagger}$ is the operator adjoint to $\mathbf{M}$, defined by

$$
\begin{gathered}
\mathbf{M}^{\dagger} \cdot \Psi^{\dagger}(\vec{r}, E, \hat{\Omega})=-\hat{\Omega} \cdot \nabla \Psi^{\dagger}(\vec{r}, E, \hat{\Omega}) \\
+\Sigma_{T}(\vec{r}, E) \Psi^{\dagger}(\vec{r}, E, \hat{\Omega}) \\
-\iint d E^{\prime} d \hat{\Omega}^{\prime} \Sigma_{S}\left(\vec{r}, E \rightarrow E^{\prime}, \hat{\Omega} \rightarrow \hat{\Omega}^{\prime}\right) \Psi^{\dagger}\left(\vec{r}, E^{\prime}, \hat{\Omega}^{\prime}\right),
\end{gathered}
$$

$S^{\dagger}(\vec{r})$ is the adjoint source, defined by

$$
S^{\dagger}(\vec{r})=\iint d E^{\prime} d \hat{\Omega}^{\prime} \frac{\chi\left(E^{\prime}\right)}{4 \pi} \Psi^{\dagger}\left(\vec{r}, E^{\prime}, \hat{\Omega}^{\prime}\right),
$$

Bell and Glasstone (1970) and others have shown that the eigenvalue $K$ in Eq. (8) is identical to the eigenvalue $K$ in Eq. (1), hence the analysis below will just use $K$, rather than $K$ and $K^{\dagger}$.

The Green's function for this problem is defined by the equation

$$
\begin{aligned}
& \mathbf{M}^{\dagger} \cdot G^{\dagger}\left(\vec{r}_{0}, E_{0}, \hat{\Omega}_{0} \rightarrow \vec{r}, E, \hat{\Omega}\right)= \\
& \quad \delta\left(\vec{r}-\vec{r}_{0}\right) \delta\left(E-E_{0}\right) \delta\left(\hat{\Omega}-\hat{\Omega}_{0}\right) .
\end{aligned}
$$

It then follows that

$$
\begin{aligned}
\Psi^{\dagger}(\vec{r}, E, \hat{\Omega})= & \frac{1}{K} \iiint d \vec{r}_{0} d E_{0} d \hat{\Omega}_{0} v \Sigma_{F}\left(\vec{r}_{0}, E_{0}\right) \\
& \cdot S^{\dagger}\left(\vec{r}_{0}\right) G^{\dagger}\left(\vec{r}_{0}, E_{0}, \hat{\Omega}_{0} \rightarrow \vec{r}, E, \hat{\Omega}\right) .
\end{aligned}
$$

Now multiply Eq. (12) by $\chi(E) / 4 \pi$ and integrate over all $E$ and $\hat{\Omega}$ :

$$
S^{\dagger}(\vec{r})=\frac{1}{K} \int d \vec{r}_{0} S^{\dagger}\left(\vec{r}_{0}\right) H^{\dagger}\left(\vec{r}_{0} \rightarrow \vec{r}\right)
$$

where the kernel $H^{\dagger}\left(\vec{r}_{0} \rightarrow \vec{r}\right)$ represents the energyangle averaged adjoint Green's function (now weighted by the final spectrum and initial fission neutron production),

$$
\begin{aligned}
H^{\dagger}\left(\vec{r}_{0} \rightarrow \vec{r}\right)=\iiint \int d E d \hat{\Omega} d E_{0} d \hat{\Omega}_{0} \frac{\chi(E)}{4 \pi} \\
\cdot v \Sigma_{F}\left(\vec{r}_{0}, E_{0}\right) G^{\dagger}\left(\vec{r}_{0}, E_{0}, \hat{\Omega}_{0} \rightarrow \vec{r}, E, \hat{\Omega}\right) .
\end{aligned}
$$

The reciprocity relation between the direct and adjoint Green's function is

$$
G^{\dagger}\left(\vec{r}_{0}, E_{0}, \hat{\Omega}_{0} \rightarrow \vec{r}, E, \hat{\Omega}\right)=G\left(\vec{r}, E, \hat{\Omega} \rightarrow \vec{r}_{0}, E_{0}, \hat{\Omega}_{0}\right)
$$

Because of the irreversible energy dependence in the neutron slowing down process, neither $G$ nor $G^{\dagger}$ is symmetric in the initial and final arguments, and Eq. (15) is the correct reciprocity relation (Bell and Glasstone (1970)). Substituting the reciprocity relation into Eq. (14) and comparing with Eq. (7) gives

$$
\begin{array}{r}
H^{\dagger}\left(\vec{r}_{0} \rightarrow \vec{r}\right)=\iiint \int d E d \hat{\Omega} d E_{0} d \hat{\Omega}_{0} \frac{\chi(E)}{4 \pi} \\
\cdot v \Sigma_{F}\left(\vec{r}_{0}, E_{0}\right) G\left(\vec{r}, E, \hat{\Omega} \rightarrow \vec{r}_{0}, E_{0}, \hat{\Omega}_{0}\right) \\
=H\left(\vec{r} \rightarrow \vec{r}_{0}\right) .
\end{array}
$$

Eq. (13) then becomes

$$
S^{\dagger}(\vec{r})=\frac{1}{K} \int d \vec{r}_{0} S^{\dagger}\left(\vec{r}_{0}\right) H\left(\vec{r} \rightarrow \vec{r}_{0}\right)
$$

Eq. (17) is an integral equation for the adjoint neutron source at $\vec{r}$ expressed in terms of the kernel $H$.

\section{II.C. Comments on the Forward and Adjoint Integral Equations for Neutron Source}

The fundamental mode eigenvalues and eigenfunctions of Eqs. (6) and (17) have been proven to exist for the continuous-energy form of the transport equation (Birkhoff and Varga (1958)). The fundamental mode eigenvalue is real, and the fundamental mode eigenfunction is non-negative. For the one-speed integral transport equation for the scalar flux derived from Eq. (1), it has been proven (Lehner (1955); Sahni (1996)) that all of the higher modes exist, with discrete real eigenvalues and real eigenfunctions. The one-speed integral equation for the scalar flux is self-adjoint due to the symmetry of the kernel in the integral equations. (In the notation used here: the kernel $H\left(\vec{r}_{0} \rightarrow \vec{r}\right)$ is equal to $H\left(\vec{r} \rightarrow \vec{r}_{0}\right)$ for a one-speed treatment; an integral operator with a symmetric kernel has an infinite set of discrete real eigenvalues and associated real eigenfunctions.) 
For the multigroup transport equation and continuous-energy transport equation, it is conventional practice to assume that higher modes exist, with real eigenvalues and eigenfunctions, even though that has not been proven. (This assumption will be demonstrated empirically for reactor-type problems, using the fission matrix and continuous-energy Monte Carlo for determining the source eigenfunctions.)

Letting $K_{n}$ denote the $n$-th eigenvalue of Eqs. (6) and (17), $S_{n}(\vec{r})$ the $n$-th eigenfunction, and $S_{n}^{\dagger}(\vec{r})$ the $n$-th adjoint eigenfunction, with $n=0$ denoting the fundamental mode and $n>0$ the higher modes,

$$
\begin{aligned}
& S_{n}(\vec{r})=\frac{1}{K_{n}} \int d \vec{r}_{0} S_{n}\left(\vec{r}_{0}\right) H\left(\vec{r}_{0} \rightarrow \vec{r}\right), \\
& S_{n}^{\dagger}(\vec{r})=\frac{1}{K_{n}} \int d \vec{r}_{0} S_{n}^{\dagger}\left(\vec{r}_{0}\right) H\left(\vec{r} \rightarrow \vec{r}_{0}\right) .
\end{aligned}
$$

The orthogonality relations for Eqs. (18) are

$$
\left(K_{p}-K_{q}\right) \int d \vec{r} S_{p}(\vec{r}) S_{q}^{\dagger}(\vec{r})=0 .
$$

Based upon the transport equation and the previous analysis, there is no basis for assuming that the forward eigenfunctions alone form an orthogonal basis set (i.e. are self-adjoint). Indeed they should not, because the energy-angle averaged Green's function $H$ is not a symmetric function of its arguments.

\section{II.D. Forward Fission Matrix Equations}

If the physical problem is segmented into $N$ spatial regions, and Eqs. (6) and (7) are then integrated over the volumes of each initial region $J$, with $\vec{r}_{0} \epsilon V_{J}$, and final region $I$, with $\vec{r} \in V_{I}$, then the following equations are obtained:

$$
S_{I}=\frac{1}{K} \sum_{J=1}^{N} F_{I, J} S_{J}
$$

where

$$
\begin{aligned}
F_{I, J} & =\int_{\vec{r} \in V_{I}} d \vec{r} \int_{\vec{r}_{0} \in V_{J}} d \vec{r}_{0} \frac{S\left(\vec{r}_{0}\right)}{S_{J}} H\left(\vec{r}_{0} \rightarrow \vec{r}\right), \\
S_{J} & =\int_{\vec{r}^{\prime} \in V_{J}} S\left(\vec{r}^{\prime}\right) d \vec{r}^{\prime} .
\end{aligned}
$$

The matrix element $F_{I, J}$ is equal to the number of fission neutrons born in the region $I$ due to one average fission neutron starting in region $J$. The matrix $\bar{F}$ is called the fission matrix. The fundamental mode eigenvalue of this matrix is formally identical to the eigenvalue $K$ in Eq. (1), and the fundamental mode eigenvector is the regionwise fission neutron source distribution. In matrix-vector form, Eq. (20) is

$$
\vec{S}=\frac{1}{K} \bar{F} \vec{S},
$$

where $\vec{S}$ is a vector of length $N$ giving the singlegeneration production of neutrons in each region from fission, and $\bar{F}$ is a full matrix of size $N \times N$. $K$ is formally identical to $k_{e f f}$, the eigenvalue of the system.

The interpretation of the $S_{I}$ terms, however, requires some discussion. Each $S_{I}$ term is the single-generation production of fission neutrons in region $V_{I}$. If the fission matrix is tallied with a converged source distribution, then discretization error is not introduced. Since tallying with an unconverged distribution is generally desired, this is usually not the case. If the regions $V_{I}$ are small enough, regardless of the source distribution used for tallying, $S_{I} / V_{I}$ accurately represents a discrete approximation to the actual source $S(\vec{r})$ from Eq. (3). The notion of "small enough" will be discussed next.

\section{II.E. Adjoint Fission Matrix Equations}

If the physical problem is segmented into $N$ spatial regions, and Eqs. (13) and (14) are then integrated over the volumes of each initial region $J$, with $\vec{r}_{0} \in V_{J}$, and final region $I$, with $\vec{r} \in V_{I}$, then the folowing equations are obtained:

$$
S_{I}^{\dagger}=\frac{1}{K} \sum_{J=1}^{N} F_{I, J}^{\dagger} S_{J}^{\dagger},
$$

where

$$
\begin{aligned}
F_{I, J}^{\dagger} & =\int_{\vec{r} \in V_{I}} d \vec{r} \int_{\vec{r}_{0} \in V_{J}} d \vec{r}_{0} \frac{S^{\dagger}\left(\vec{r}_{0}\right)}{S_{J}^{\dagger}} H\left(\vec{r} \rightarrow \vec{r}_{0}\right), \\
S_{J}^{\dagger} & =\int_{\vec{r}^{\prime} \in V_{J}} S^{\dagger}\left(\vec{r}^{\prime}\right) d \vec{r}^{\prime} .
\end{aligned}
$$

In matrix-vector form, Eq. (23) is

$$
\vec{S}^{\dagger}=\frac{1}{K} \bar{F}^{\dagger} \vec{S}^{\dagger}
$$

The components of the adjoint vector $\vec{S}^{\dagger}$ are the importances to neutron multiplication of the fission neutrons born each region $I$. Equations (23-25) are the adjoint form of Eqs. (20-22). The eigenvalue $K$ of these equations is formally identical to that in Eq. (1) and Eq. (8).

\section{II.F. Relationship Between the Forward and Adjoint Fis-} sion Matrix

Comparison of the matrices $\bar{F}$ and $\bar{F}^{\dagger}$ is now performed by examining the fission matrix elements for 
the direct and adjoint matrices for a particular $(I, J)$ and $(J, I)$, respectively. Recalling Eqs. (21) and (24), but interchanging the integration variables of Eq. (24) to compare $F_{I, J}$ with $F_{J, I}^{\dagger}$ gives:

$$
\begin{aligned}
F_{I, J} & =\int_{\vec{r} \in V_{I}} d \vec{r} \int_{\vec{r}_{0} \in V_{J}} d \vec{r}_{0} \frac{S\left(\vec{r}_{0}\right)}{S_{J}} \iiint \int d E d \hat{\Omega} d E_{0} d \hat{\Omega}_{0} \\
& \cdot v \Sigma_{F}(\vec{r}, E) \frac{\chi\left(E_{0}\right)}{4 \pi} G\left(\vec{r}_{0}, E_{0}, \hat{\Omega}_{0} \rightarrow \vec{r}, E, \hat{\Omega}\right), \quad(26 \mathrm{a}) \\
F_{J, I}^{\dagger} & =\int_{\vec{r}_{0} \in V_{J}} d \vec{r}_{0} \int_{\vec{r} \in V_{I}} d \vec{r} \frac{S^{\dagger}(\vec{r})}{S_{I}^{\dagger}} \iiint \int d E d \hat{\Omega} d E_{0} d \hat{\Omega}_{0} \\
& \cdot v \Sigma_{F}(\vec{r}, E) \frac{\chi\left(E_{0}\right)}{4 \pi} G\left(\vec{r}_{0}, E_{0}, \hat{\Omega}_{0} \rightarrow \vec{r}, E, \hat{\Omega}\right) . \quad(26 \mathrm{~b})
\end{aligned}
$$

While the Green's functions and physical data in the energy-angle integrations are identical, the spatial weighting functions differ in Eqs. (26a) and (26b). For Eq. (26a), the spatial weighting function is $S(\vec{r}) / S_{J}$, while for Eq. (26b) the spatial weighting function is $S^{\dagger}(\vec{r}) / S_{I}$. If the spatial mesh used for the fission matrix is fine enough so that

$$
\begin{aligned}
& \frac{S^{\dagger}(\vec{r})}{S_{I}^{\dagger} / V_{I}} \simeq 1 \text { for } \vec{r} \in V_{I}, \\
& \frac{S\left(\vec{r}_{0}\right)}{S_{J} / V_{J}} \simeq 1 \text { for } \vec{r} \in V_{J},
\end{aligned}
$$

then Eqs. (26a) and (26b) become identical, and $F_{I, J}=$ $F_{J, I}^{\dagger}$. That is, in the limit of a fine enough spatial mesh, the adjoint fission matrix is equal to the transpose of the (direct) fission matrix,

$$
\bar{F}^{\dagger}=\bar{F}^{T},
$$

so that the fission matrix equations are consistent with the continuous formulation given by Eq. (16).

\section{II.G. Basis for Higher Eigenmode Analysis Using the Fission Matrix}

Eqs. (6) and (13) are specific to the fundamental modes of the forward and adjoint $K$-eigenvalue transport equation for continuous-energy problems. Higher modes have always been assumed to exist for Eqs. (6) and (13). For the discretized, region-integrated Eqs. (20) and (23) for the fission matrix approach, higher eigenmodes can be determined according to:

$$
\begin{aligned}
& \vec{S}_{n}=\frac{1}{K_{n}} \bar{F} \vec{S}_{n}, \\
& \vec{S}_{n}^{\dagger}=\frac{1}{K_{n}} \bar{F}^{T} \vec{S}_{n}^{\dagger}, \quad n=0,1, \ldots, N \\
& K_{0}>\left|K_{1}\right|>\left|K_{2}\right|>\ldots>\left|K_{N}\right|,
\end{aligned}
$$

where the subscript $n$ refers to the mode, with $n=0$ the fundamental mode. In Eqs. (29), the adjoint fission matrix has been replaced by the transpose of the direct fission matrix, according to the discussion regarding Eq. (28). For a problem with $N$ regions in the mesh for the fission matrix, $\bar{F}$ is an $N \times N$ matrix with $N$ discrete eigenvalues. Because $\bar{F}$ is a nonsymmetric matrix, the eigenvalues and eigenvectors may be complex, although the fundamental mode must be strictly real.

The transport equation is biorthogonal and may or may not be orthogonal as well; different eigenvectors $\vec{S}_{p}$ and $\vec{S}_{q}$ (and also $\vec{S}_{p}^{\dagger}$ and $\vec{S}_{q}^{\dagger}$ ) need not be orthogonal to each other. The forward and adjoint eigenvectors, however, are orthogonal:

$$
\left(K_{p}-K_{q}\right)\left(\vec{S}_{q}^{\dagger} \cdot \vec{S}_{p}\right)=0
$$

The adjoint eigenvectors $\vec{S}_{q}^{\dagger}$ can be interpreted as the left eigenvectors of the matrix $\bar{F}$, while the forward eigenvectors $\vec{S}_{p}$ are the right eigenvectors of the matrix $\bar{F}$. It is a mathematical identity that the left and right eigenvectors are orthogonal.

\section{II.H. Monte Carlo Estimation and Storage of Fission Matrix}

As noted in section II.A, the fission matrix elements defined rigorously by Eqs. (26) may be estimated by continuous-energy Monte Carlo methods. For right eigenvector calculation, if a coarse mesh is used to define the spatial regions for tallying Eq. (26a), then the tallies cannot be made until after the fission source distribution has converged, since the spatial weighting functions correspond to the stationary source distribution. However, if a fine enough mesh is used such that Eq. (27) is valid, then Eqs. (26) become independent of the spatial weighting functions, and valid tallies can be made even before the source distribution converges, for both left and right eigenvector calculation. In practice, fission matrix tallies here are chosen to begin at the third iteration cycle, and to accumulate over all subsequent cycles. (Single-cycle estimates of the fission matrix are never used.) The first two cycles are skipped to reduce discretization error, which is largest in the proximity of the initial distribution where no powering out of higher modes has occured. In section III.A, studies of the convergence of fission matrix results with mesh refinement are used to demonstrate the validity of this overall approach.

The choice of region shapes and sizes for determining the fission matrix is arbitrary, as long as all fissionable regions in the physical problem are covered. For the initial testing in MCNP6, a simple, uniform, 3D, Cartesian 
mesh is used, with different numbers of mesh intervals permitted in the $\mathrm{x}-, \mathrm{y}-$, and $\mathrm{z}$-directions. The mesh overlays the detailed Monte Carlo geometry for the physical problem and must encompass all fissionable regions in the problem. The choice of mesh for tallying the fission matrix does not affect the ordinary Monte Carlo tracking. Using a uniform mesh permits quick determination of the $i j k$ spatial mesh indices for tallies of the source at $x, y, z$.

While the initial guess for the fission neutron source distribution is arbitrary for criticality calculations, the use of a uniform volumetric source in fissionable regions is the best choice for fission matrix estimation. This aids in a complete sampling of fissionable material for fission matrix tallies in the intitial stages of the power iteration process used in MCNP6. However, in problems with a significant degree of nonuniformity of fissionable material, issues involving insufficient sampling are possible.

\section{II.I. Sparse Fission Matrix Representation}

Sparse fission matrix storage, previously entailing allocating a certain number of matrix bands ad hoc, has now been changed to a compressed-row storage scheme. With the scheme, no approximations are made; the sparsity is general, and all tallies are recorded. If the fission matrix elements are tallied for a regular 3D spatial mesh with $N=N_{x} \times N_{y} \times N_{z}$ mesh cells, then a fission neutron starting from mesh cell $[i, j, k]_{S}$ that creates a next-generation fission neutron in mesh cell $[i, j, k]_{T}$ would be tallied in the matrix tally bin $F(I, J)$, where $J=i_{S}+\left(j_{S}-1\right) N_{x}+\left(k_{S}-1\right) N_{x} N_{y}$ and $I=i_{T}+\left(j_{T}-\right.$ 1) $N_{x}+\left(k_{T}-1\right) N_{x} N_{y}$. Only the nonzero $F(I, J)$ entries are stored. In the compressed-row scheme illustrated in Fig. 1, the $L(I)$ array entries point to the start of a list of $J$ indices and corresponding nonzero $F(I, J)$ tallies. To look up the tally for $F(I, J)$, it is necessary to search the $J$ array from location $L(I)$ through $L(I+1)-1$ for the desired $J$ (if it exists), and then retrieve the corresponding $F$ tally. If the $F(I, J)$ entry is not already stored, then it must be inserted during the tallying. The size of $J$ and $F$ arrays thus increases as more neutrons are simulated.

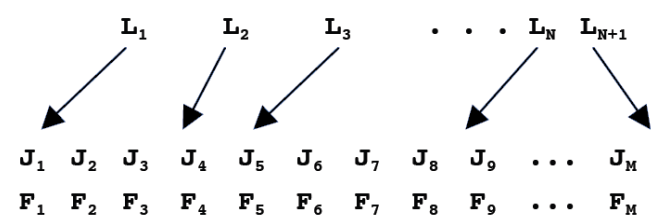

Figure 1: Compressed Row Storage
If $M$ is the total number of nonzero $F(I, J)$ tally entries, then the $L$ array has $N+1$ entries, and the $J$ and $F$ arrays each have $M$ entries. In practice, $M<<N^{2}$, resulting in very large reductions in the memory storage for the $F(I, J)$ tallies. Figure 2 shows a typical structure of the fission matrix for 2D PWR problem. The white portions of the plot correspond to zero $F(I, J)$ entries that are not stored in the compressed-row storage scheme.

The use of a sparse storage scheme had required the impementation of numerous new Monte Carlo computational algorithms, such as performing efficient tallies into the sparse matrix, eigensolvers for both the left and right eigenvectors of a general sparse matrix based on power iteration, Hotelling deflation of the solution space for the sparse power iteration to compute higher-mode left and right eigenvectors, etc. Coding was developed to perform the tallies in an efficient manner, so that the fission matrix tallies typically require less that a second of wall-clock time at the end of each batch in the Monte Carlo simulation. Total MCNP6 running time was found to not increase more than $1 \%$ due to the extra operations required for tallying the fission matrix.

In practice, MCNP6 simulations are run using a very fine spatial mesh for the fission matrix tallies, typically $1000 \times 1000$ mesh cells for $2 \mathrm{D}$ problems or $1000 \times 1000 \times$ 1000 mesh cells for 3D problems. After the MCNP6 runs have finished, the fission matrix tallies are aggregated into a more compact matrix by combining a moderate number of entries for neighboring spatial mesh cells. This aggregation results in reduced statistical uncertainties in the fission matrix elements, but potentially increases discretization error. In addition, by aggregat-

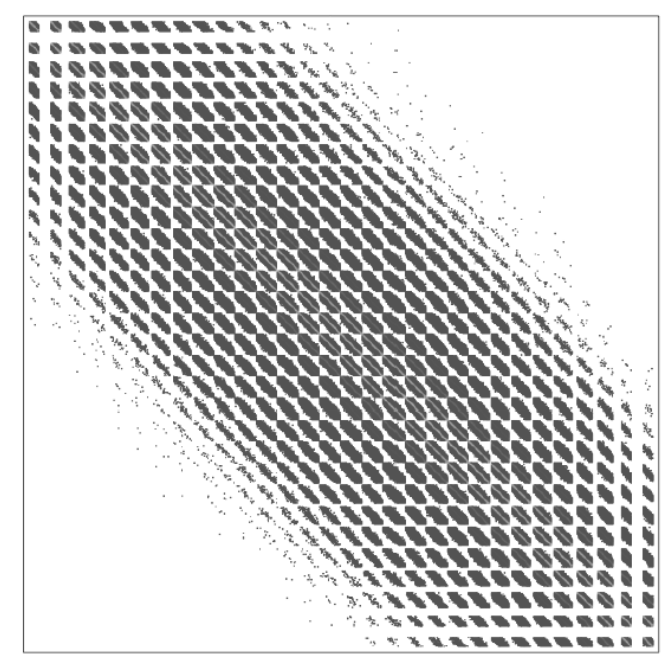

Figure 2: Fission matrix structure for 2D PWR 
ing the matrix several times and finding the eigenvalue spectrum each time, it is straightforward to assess the degree of spatial mesh detail that is necessary for a converged spectrum. This could be automated given a desired number of modes and a numerical tolerance by the user, since predictable convergence behavior is seen for all cases studied. Memory restrictions would limit this tolerance; large and highly-coupled problems could make this limit too restrictive.

For Monte Carlo codes that use a fixed number of starting source neutrons for every cycle, the region tallies are simply incremented by one for each neutron; for MCNP6 with a varying number of neutrons starting each cycle, the tallies need to be incremented by $M_{0} / M$, where $M_{0}$ is the number of neutrons starting in the initial cycle, and $M$ is the number that started the current cycle.

This approach eliminates any inter-process communication overhead during MPI parallel processing, since the entire fission matrix estimation can be performed on the master node, using only the existing "fission bank" information. (If fission matrix tallies were instead made during the neutron random walks, for instance using a path-length estimator, then the entire set of fission matrix tallies would then need to be accumulated across all nodes, which could involve many GB of MPI messagepassing at considerable overhead cost.) Path-length estimation of the fission matrix was found to approach the same expectation faster than point-to-point tallies, but not to an extent to compensate for the increased computational overhead.

\section{II.J. Matrix Higher Eigenmode Calcuation}

At any desired cycle, the numerator-only $F_{I, J}$ tallies may be normalized by dividing by the total accumulated source in the starting regions (i.e., $J$ index) to form the normalized (i.e., actual) fission matrix. Then the eigenvalues and eigenvectors of the fission matrix may be found. Four different solving methods were evaluated and verified against each other: standard power iteration with Hotelling deflation, a direct non-symmetric eigensolver (for smaller fission matrices), an iterative solver using the implicitly restarted Arnoldi method (IRAM), and the nonsymmetric eigensolver available in MATLAB (Mathworks (2013)). The IRAM solver is fastest for general use (Josey and Veit (2013)) and is well suited to sparse matrix problems. It requires only the multiplication of the sparse matrix times a vector, and it does not require storage of the full matrix. Power iteration is robust but slow. The direct solver and MATLAB solver require storage of the full fission matrix, hence can only be used for problems with a small fission matrix (i.e., very coarse spatial resolution). For practical application, the fission matrix tallies could be accumulated for all problem iterations, and then the fission matrix eigenvalues and eigenvectors could be determined only after the Monte Carlo calculation had completed. If it is desired to obtain the fission matrix solution during the Monte Carlo calculation, to potentially use it to accelerate the overall source convergence, then the fundamental mode eigenvector could be determined at the end of any cycle.

\section{II.K. Higher-mode Forward Flux Calculation}

The right eigenvectors of the fission matrix can be used to calculate higher-mode neutron fluxes and any desired reaction rates. This requires running a fixedsource calculation on $S_{n}(\vec{r})$,

$$
\Psi_{n}(\vec{r}, E, \hat{\Omega})=\frac{1}{K_{n}} \mathbf{M}^{-1} \cdot \frac{\chi(E)}{4 \pi} S_{n}(\vec{r}) .
$$

As both flux and fission source modes can be positive or negative for $n>0$, a flag is used internally in MCNP6 to mark the sign of a neutron's weight. That is, source neutrons are started according to the magnitude of the higher mode source and are flagged as either positive or negative. Source points with constant weight are sampled from the loaded fission source mode (starting locations are sampled until fissionable material is found). Fission is treated as capture, in accordance with the definition of the net-loss operator. An equivalent and possibly more efficient method is to contain all desired modal information in each particle history. This entails sampling source points uniformly throughout the problem's fissionable regions, and attaching a vector of tally multipliers to each source particle. Each multiplier in the vector would correspond to the magnitude of each mode at the starting location.

A concern here, examined in Section III.C, is that for greater positive/negative oscillation in the source as mode number rises, there will be more score cancellation in tallies. This may lead to larger tally relative variances than is manageable.

\section{II.L. Modal Expansion of Fission Source}

In a criticality calculation, expanding the fission bank distribution of cycle $c, f^{c}(\vec{r})$, with the fission source eigenvectors $S_{n}(\vec{r})$ can give insight into the power iteration process. Yamamoto et. al. (2013) have shown this by using eigenmodes calculated with the method of characteristics. The modal expansion coefficients $a_{n}^{c}$, found using biorthogonality [Eqs. (32)], indicate the degree of excitation of different eigenmodes. Typically 
convergence is only characterized by the dominance ratio, $K_{1} / K_{0}$, but this expansion,

$$
\begin{aligned}
& f^{c}(\vec{r})=\sum_{n=0}^{\hat{N}} a_{n}^{c} S_{n}(\vec{r}), \\
& a_{n}^{c}=\frac{\left\langle f^{c}(\vec{r}), S_{n}^{\dagger}(\vec{r})\right\rangle}{\left\langle S_{n}(\vec{r}), S_{n}^{\dagger}(\vec{r})\right\rangle},
\end{aligned}
$$

gives further information. ( $\hat{N}$ is a maximum mode number of interest, less than the total number of modes.) To calculate $a_{n}^{c}$, left and right eigenvectors of a tallied fission matrix are calculated. These are then used for the inter-cycle calculation of Eq. (32b). Different initial distribution will excite different modes, as shown in Section III.E.

Recalling the basic proof of power iteration, which assumes linearly independent eigenvectors (the case here), the decay rate of mode $n>0$ is given by $K_{n} / K_{0}$. Similar rates for the expansion coefficients here would be expected.

\section{Applications}

The fission matrix capability was implemented in a research version of MCNP6 with the sparse matrix tally scheme. Testing was performed on a variety of reactor applications. All results described below were obtained with ENDF/B-VII.0 continuous-energy data and continuous-energy physics (Chadwick et. al. (2006)). Fission matrices were accumulated during standard criticality calculations, beginning with the third cycle to ameliorate discretization error, which is largest in the initial cycles. The third cycle was found to be suitable by observing the correlation between cycle-wise fission matrix results and cycle number become negligible by the third cycle, for a simple test problem with a coarse mesh. For a 2D reactor model, properties of the eigenvalue spectrum (Sections III.A-III.B) and eigenmodes (Sections III.C) are examined, followed by a demonstration of source convergence acceleration for a fuel storage vault problem (Section III.D). Eigenmode expansion of the iterated fission source for the $2 \mathrm{D}$ reactor problem is then shown in Section III.E.

\section{III.A. Eigenvalue Spectrum Convergence with Mesh Re-} finement

When the fission matrix is estimated during a continuous-energy Monte Carlo $K$-effective calculation, the $N$ eigenvalues of the $N \times N$ fission matrix may be determined, where $N$ is the number of fissionable regions

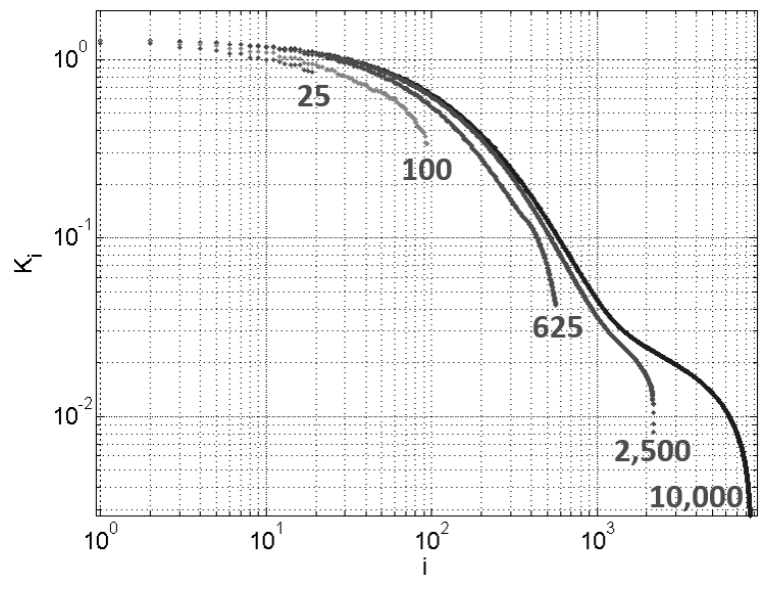

Figure 3: Eigenvalue spectrum for whole-core 2D PWR model, with explicit geometry, ENDF/B-VII.0 crosssections, and continuous-energy physics. Real part of $K_{i}$ is shown for various spatial mesh resolutions.

in the spatial mesh used for tallying the fission matrix. If the calculation is repeated using finer mesh resolution, i.e., higher $N$, the eigenvalue spectrum appears to have converged to its limiting distribution such that further mesh refinement does not alter it. Convergence of the eigenvalue spectrum provides direct evidence that the spatial mesh resolution is fine enough that Eq. (27) is valid. Figure 3 provides an example of a mesh refinement study for the eigenvalue spectrum for a whole-core 2D PWR model with explicit geometry (Nakagawa and Mori (1993)). Fission matrix tallies were accumulated over 298 cycles of $500 \mathrm{k}$ neutrons each. As the mesh is refined (larger $N$, smaller region size), the first few 100 eigenvalues converge.

Figure 4 shows the detailed convergence with mesh refinement of the first 15 eigenvalues for this model, all calculated from the same $1.49 \times 10^{8}$ histories. For this problem, it appears that the $100 \times 100$ mesh provides satisfactory convergence for the 15 modes shown. It appears, overall, that higher mode numbers require finer meshing for convergence. This can be understood in terms of the growing inapplicability of the fundamentalmode spatial weighting function arising from the simulation, and the increasingly oscillatory higher-mode spatial weighting functions. These mesh refinement studies demonstrate that the eigenvalue spectrum converges smoothly to a stationary discrete distribution. Further mesh refinement does not change the spectrum of lower eigenvalues, indicating that the fission matrix results have converged to the limiting values of the fully-continuous form of the transport equation. 


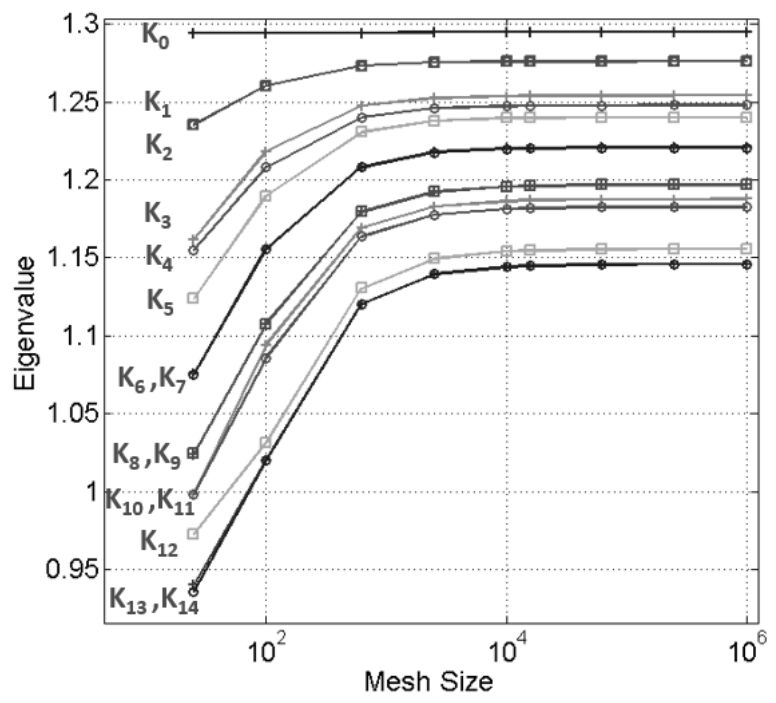

Figure 4: Convergence of the first 15 eigenvalues for whole-core 2D PWR model as $N$ is increased. Same $1.49 \times 10^{8}$ histories used for each relevant fission matrix.

\section{III.B. Real vs. Complex Eigenvalues}

Figure 5 shows the imaginary component of the eigenvalue spectrum of the aforementationed 2D PWR problem. Only positive imaginary components are shown; not shown are the negative components of the

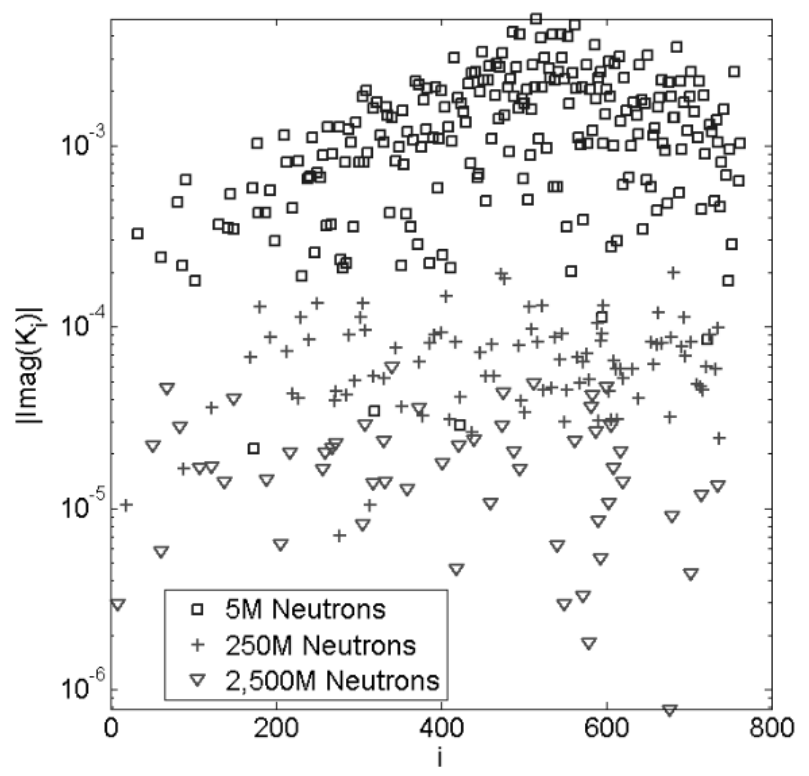

Figure 5: Detail for positive imaginary parts of 2D PWR eigenvalues, after 5M, 250M, and 2500M neutrons. complex conjugates. The fission matrix was tallied on a $30 \times 30 \mathrm{mesh}$, and spectra are shown for matrices tallied from 5M, 250M, and 2500M neutrons. As more neutrons are followed, the imaginary parts of the eigenvalues become very much smaller: for the three tally sizes shown here, the average nonzero imaginary components differ by approximately an order of magnitude and become fewer in number. Because the fission matrix is nonsymmetric and each of the matrix elements has associated statistical uncertainty, the numerical solutions can give rise to imaginary components. However, behavior due to reduced uncertainty provides strong evidence (but not proof) that all of the $K$-eigenvalues are discrete and real-valued for reactor-type problems.

\section{III.C. Fission Source and Flux Eigenmodes}

A fission matrix was generated for the 2D PWR problem using a $50 \times 50$ spatial mesh, 300 batches (skipping the initial 2) and $500 \mathrm{~K}$ neutrons/batch. Then the first 30 eigenmodes of the fission matrix were found, shown in Fig. 6. Each of these 30 eigenmodes for the fission neutron source was then used as the source for fixed source calculations to determine fluxes. The 30 fixed source calculations each used $500 \mathrm{~K}$ neutron histories; Fig. 7 gives results for the flux and relative uncertainties. Each of these fixed source calculations required only about one minute of MCNP6 time using 8 threads. Above-thermal $(0.625 \mathrm{eV}-20 \mathrm{MeV})$ flux modes are very similar to the fission source modes, and the most notable feature of the thermal $(0-0.625 \mathrm{eV})$ flux modes are the peaks in the peripheral water moderator. The relative uncertainty plots gives a favorable result: the high relative uncertainties are localized near the zero-contour lines. Uncertainties are manageable where the functions are significantly nonzero, notwithstanding the cancellation of tallies.

Inner products of forward and adjoint fission source modes were calculated to verify biorthogonality. The nearness to orthogonality of the forward modes was then examined. Among the first 30 modes, most inner products were $0.001 \%-0.1 \%$ of the normalized value of unity for the inner products of identical modes. But, some inner products were $1 \%-10 \%$ of unity: particularly inner products of modes with near-multiplicity. Modes with near-mulitplicity have nearby eigenvalues and similar but rotated eigenmode shapes. Incidentally, these modes were found to be the most sensitive to statistical uncertainty and mesh size. These results confirm that while forward modes alone are not orthogonal, it is a reasonable assumption for reactor analysis. 


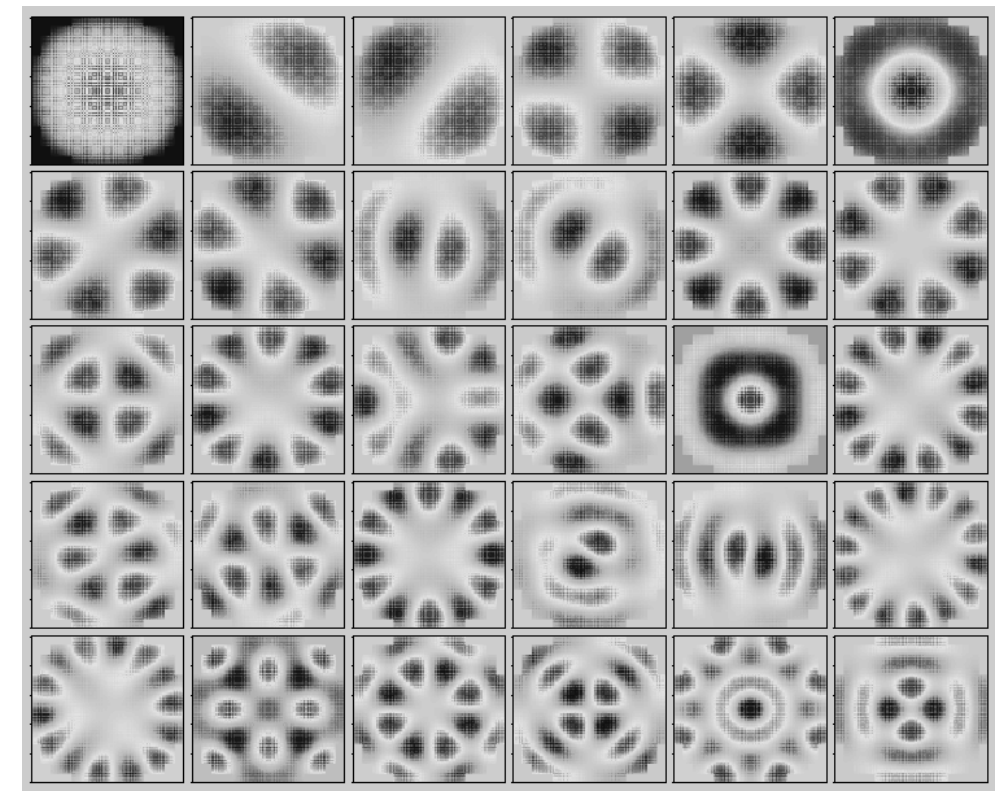

Figure 6: First 30 fission source modes of 2D PWR on a $100 \times 100$ mesh.
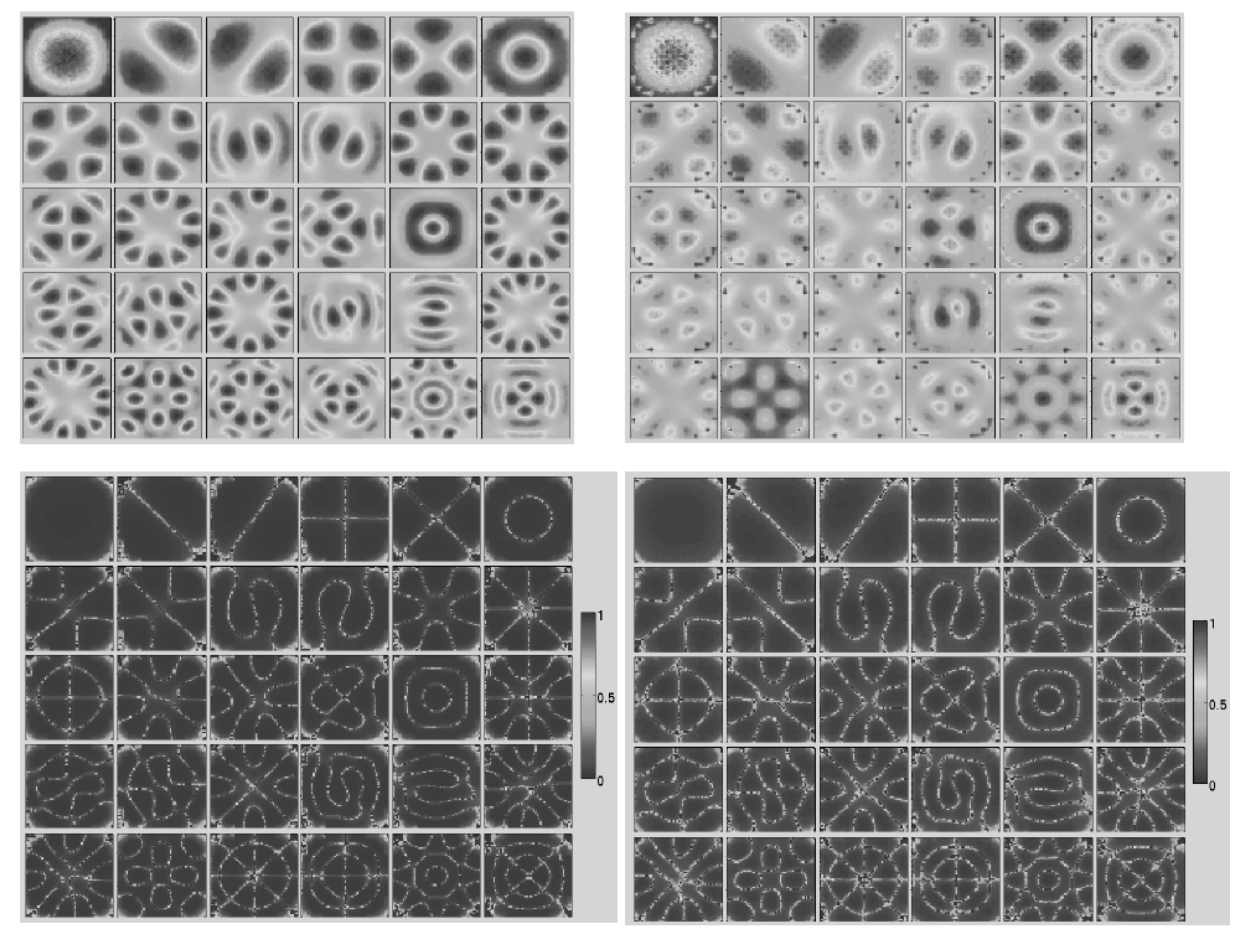

Figure 7: First 30 forward flux modes (top) of 2D PWR and relative uncertainties (bottom), for 0-0.625 eV (left) and $0.625 \mathrm{eV}-20 \mathrm{MeV}$ (right) 


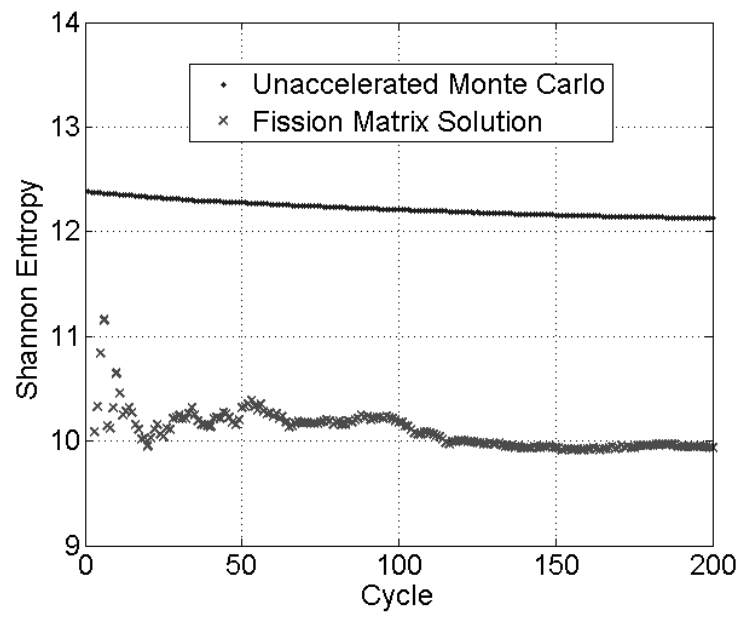

Figure 8: Vault model: Shannon entropy to diagnose source convergence for standard MCNP6 and the fission matrix approach.

\section{III.D. Fuel Storage Vault: Convergence Acceleration}

The fundamental-mode fission source calculated from the fission matrix is of a lower spatial order than the standard Monte Carlo solution, and it is calculated from cumulatively-tallied results. Consequently, the convergence of the Monte Carlo fission source can be accelerated by replicating the fission matrix solution in the Monte Carlo fission site distribution after any given cycle. This is performed in MCNP6 by the duplication and deletion of fission sites existing at the end of any cycle.

Benchmark Problem 1 from the OECD/NEA Source Convergence Benchmark (Smith et. al. (2001)) is examined here to demonstrate the proficiency of acceleration with the fission matrix. The problem contains 36 large, loosely-coupled spent fuel assemblies in water surrounded by concrete reflector. A sole assembly has concrete reflector on two sides, as opposed to one or zero for the others. Consequently, this single assembly is by far the most reactive, with a total fission rate over a factor of 10,000 greater that the least reactive assembly. Unaccelerated power iteration with an initial flat distribution requires around 2000 cycles for fission source convergence. The slow convergence rate is indicated by the large dominance ratio calculated from the fission matrix: 0.997.

Figure 8 shows the convergence behavior of the standard MCNP6 and fission matrix results. The fission matrix is tallied for cycles 3-200, with a batch size of $1 \mathrm{M}$. The spatial mesh is $96 \times 12 \times 10$, corresponding in the $\mathrm{x}-\mathrm{y}$ plane to sixteen mesh regions for every assembly. By cycle 30, the fission matrix gives a reasonable converged fundamental eigenvector. Convergence of unaccelerated Monte Carlo very much follows the deterministic behavior of a matrix eigenvalue calculation, the rate given by the dominance ratio. In the case of the fission matrix, convergence is achieved by better estimation of matrix elements before solving for the fundamental mode, thus no discernable pattern emerges.

\section{III.E. Expansion Coefficients}

For the 2D PWR problem, cycle-wise eigenmode expansion coefficients of Eq. (32b) were calculated. Results from two initial guesses are shown here: a point in the center of the upper-right quadrant of the reactor (Fig. 9) and a point in the center of the reactor (Fig. 10). Modes are numbered in the same manner as the order of Fig. 6. In these plots, important modes are labeled; the center guess excites radially-symmetric modes that have center-peaks and the corner guess excites asymmetric modes that have peaks in the relevant corner (a flat guess, a uniform circle, was also examined, and was found to excite radially-symmetric modes, but to a much lesser extent than the center-point case). The higher the mode number, the quicker it decays away as power iteration proceeds. Tables I and II show the proximity of the expected values to the values found from doing a least squares fit of the coefficients while significantly nonzero.

There are other observations that can be made regarding the expansion coefficients once they have converged. As lower mode numbers have the higher eigen-

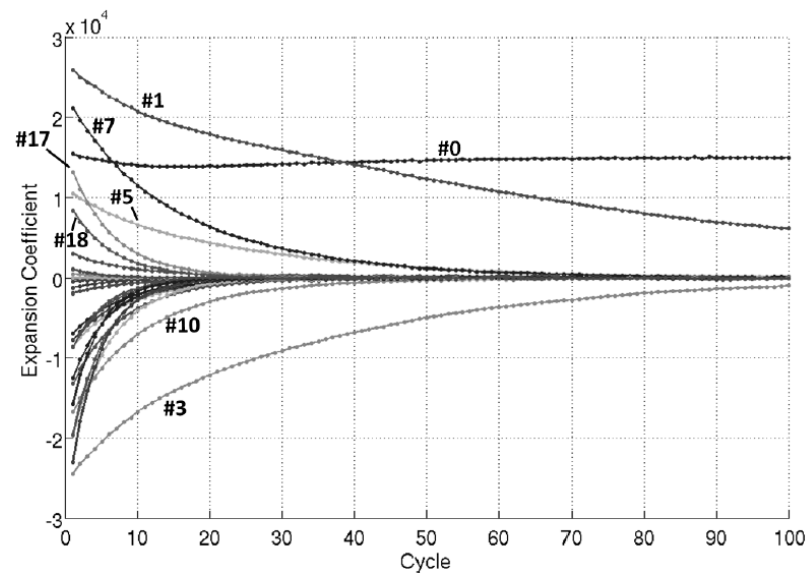

Figure 9: First 30 eigenmode expansion coefficients for 2D PWR; initial guess is the center point of the top-right quadrant of the core. 


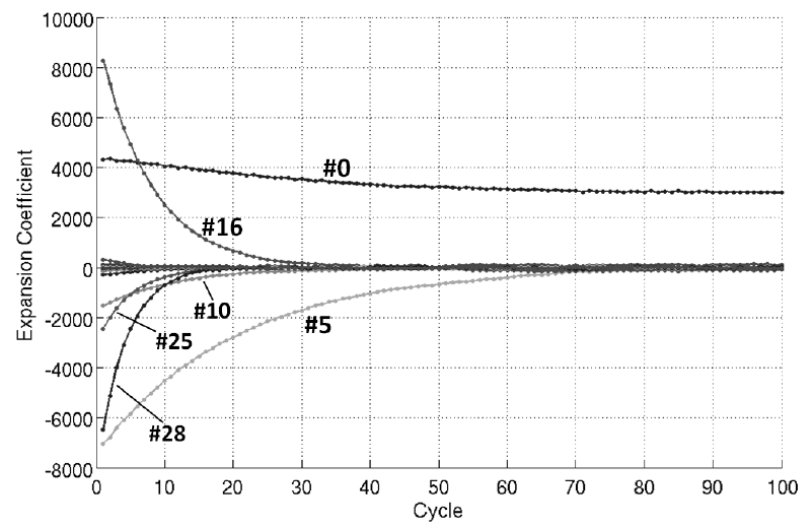

Figure 10: First 30 eigenmode expansion coefficients for 2D PWR; initial guess is the center point of the reactor.

Table I: Empirical power decay rates of expansion coefficients for the center-point initial guess run (batch size $=1 \mathrm{M}$ ), and relevant eigenvalue ratios.

\begin{tabular}{c|c|c|c|c}
\hline $\begin{array}{c}\text { Mode } \\
\#\end{array}$ & $\begin{array}{c}\text { Cycle } \\
\text { Range for } \\
\text { Fit }\end{array}$ & $\begin{array}{c}\text { Empirical } \\
\text { Decay } \\
\text { Rate }\end{array}$ & $K_{i} / K_{0}$ & $C / E$ \\
\hline 5 & $1-80$ & 0.95371 & 0.95757 & 0.99597 \\
10 & $1-30$ & 0.90994 & 0.91655 & 0.99279 \\
16 & $1-30$ & 0.87385 & 0.87954 & 0.99354 \\
25 & $1-15$ & 0.80733 & 0.81315 & 0.99283 \\
18 & $1-20$ & 0.78111 & 0.78421 & 0.99605 \\
\hline
\end{tabular}

values, eigenmodes of higher number should be less correlated from cycle to cycle. Though not shown here, a look at these these coefficients reveals significant lag correlation in the drift of mode 2, whereas mode 29 has a much tighter and uncorrelated oscillation about the value of zero. The lag-correlation coefficients were directly evaluated for these expansion coefficients, and some evidence of relation to the eigenvalue spectrum was found, as assumed in MacMillan (1973).

\section{SUMMARY AND FUTURE WORK}

Theoretical and practical work on the fission matrix method is discussed here. The fission matrix, tallied without significant extra cost during a Monte Carlo criticality calculation, consists of spatial transition terms between two fission generations. With a sufficiently refined mesh (achieved here with a sparse storage scheme) these rates can be accurately tallied without knowledge of the correct fission distribution. The matrix can then be solved for $K$-eigenvalues and eigenvectors. The right
Table II: Empirical power decay rates of expansion coefficients for the corner-point initial guess run (batch size $=500 \mathrm{~K}$ ), and relevant eigenvalue ratios.

\begin{tabular}{c|c|c|c|c}
\hline $\begin{array}{c}\text { Mode } \\
\#\end{array}$ & $\begin{array}{c}\text { Cycle } \\
\text { Range for } \\
\text { Fit }\end{array}$ & $\begin{array}{c}\text { Empirical } \\
\text { Decay } \\
\text { Rate }\end{array}$ & $K_{i} / K_{0}$ & $C / E$ \\
\hline 1 & $1-150$ & 0.98609 & 0.98530 & 1.00080 \\
3 & $1-100$ & 0.96945 & 0.96756 & 1.00195 \\
5 & $1-50$ & 0.95810 & 0.95619 & 1.00199 \\
7 & $1-50$ & 0.94412 & 0.94058 & 1.00377 \\
8 & $1-15$ & 0.90134 & 0.92119 & 0.98822 \\
9 & $1-20$ & 0.89848 & 0.92118 & 0.97535 \\
10 & $1-45$ & 0.92062 & 0.91388 & 1.00737 \\
\hline
\end{tabular}

and left eigenvectors correspond to forward and adjoint fission source modes, respectively.

Eigenvalues calculated from the fission matrix are shown to stably converge for an increasing mesh resolution. Imaginary components are shown to decrease as statistical uncertainty is reduced. Results from both higher mode fission souce and flux calculations are shown. Fission source results are shown to both significantly accelerate source convergence in a criticality calculation, and better characterize unaccelerated convergence.

The weighting of higher-mode adjoint fluxes is necessary for practical applications such as second-order perturbation theory and quasi-static transient calculations. This weighting can be performed in the same manner as the CLUTCH (Contribution-Linked eigenvalue sensitivity/Uncertainty estimation via Tracklength importance CHaracterization) method (Perfetti and Rearden (2013)), but with the use of higher-mode adjoint sources. Work is also being done to improve the robustness of the source convergence acceleration method, though the use of a high-order/low-order matrix that maintains total consistency with the Monte Carlo simulation.

\section{Acknowledgements}

This work was supported by the U.S. Department of Energy NNSA Advanced Simulation and Computing Program and Nuclear Criticality Safety Program, and the DOE Nuclear Energy University Programs.

\section{References}

K.W. MORTON, "Criticality Calculations by Monte Carlo Methods", United Kingdom Atomic Energy Research Establishment, Harwell, Report T/R-1903 (1956). 
E.L. KAPLAN, "Monte Carlo Methods for Equilibrium Solutions in Neutron Multiplication", Lawrence Radiation Laboratory, UCRL5275-T (1958).

J.M. HAMMERSELY and D.C. HANDSCOMB Monte Carlo Method, Chapter 8, Methuen \& Co. (1964).

T. URBATSCH, "Fission Matrix Capabilites in MCNP", TJU-92-557 (1992).

T. KITADA and T. TAKEDA, "Effective Convergence of Fission Source Distribution in Monte Carlo Simulation", J. Nucl. Sci. Technol., 38, 5, 324-329 (2001).

J. DUFEK and W. GUDOWSKI, "Fission Matrix Based Monte Carlo Criticality Calculations”, Ann. Nucl. Energy, 36, 1270-1275 (2009).

M. WENNER and A. HAGHIGHAT, "A Fission Matrix Based Methodology for Achieving an Unbiased Soluation for Eigenvalue Monte Carlo Simulations", Prog. Nucl. Sci. Tech., 2, 886-892 (2011).

J.T. GOORLEY, et. al., "Initial MCNP6 Release Overview-MCNP6 version 1.0”, LA-UR-13-22934, Los Alamos National Laboratory (2013).

S.E. CARNEY, F.B. BROWN, B.C. KIEDROWSKI, and W.R. MARTIN, "Fission Matrix Capability for MCNP Monte Carlo", Trans. ANS, 107 (2012).

F.B. BROWN, S.E. CARNEY, B.C. KIEDROWSKI, and W.R. MARTIN "Fission Matrix Capability for MCNP, Part I - Theory", Proc. $M \mathcal{E} C$ 2013, Sun Valley, ID, May 5-9 2013, on CD-ROM.

S.E. CARNEY, F.B. BROWN, B.C. KIEDROWSKI, and W.R. MARTIN "Fission Matrix Capability for MCNP, Part II - Applications", Proc. ME C 2013, Sun Valley, ID, May 5-9 2013, on CD-ROM.

S.E. CARNEY, F.B. BROWN, B.C. KIEDROWSKI, and W.R. MARTIN "Higher-Mode Applications of Fission Matrix Capability for MCNP", LA-UR-13-26615 (2013).

G.I. BELL and S. GLASSTONE, Nuclear Reactor Theory, Van Nostrand Reinhold (1970).

G. BIRKHOFF and R.S. VARGA, "Reactor Criticality and Nonnegative Matrices", J. Soc. Indust. Appl. Math., 6, 4, 354-377 (1958).

J. LEHNER and G.M. WING, Pure Appl. Math., VIII, 217 (1955).

D.C. SAHNI, "Some New Results Pertaining to Criticality and Time Eigenvalues of One-Speed Neutron Transport Equation”, Prog. Nuc. Energy, 30, 3, 305-320 (1996).

MATLAB 8.0, The MathWorks, Inc., Natick, Massachusetts, United States.

C. JOSEY and M.D. VEIT, "Eigenfunction Decomposition of Reactor Perturbations and Transitions Using MCNP Monte Carlo", LAUR-13-26449 (2013).

A. YAMAMOTO, K. SAKATA, and T. ENDO, "Explicit Estimation of Higher Order Modes in Fission Source Distribution of MonteCarlo Calculation", Proc. MEEC 2013, Sun Valley, ID, May 5-9 2013, on CD-ROM.

M.B. CHADWICK, et. al. "ENDF/B-VII.0: Next Generation Evaluated Nuclear Data Library for Nuclear Science and Technology", Nuclear Data Sheets, 107, pp. 2931-3060 (2006).

M. NAKAGAWA and T. MORI, "Whole Core Calculations of Power Reactors by use of Monte Carlo Method", J. Nuc. Sci. Technol., 30 [7], pp. 692-701 (1993).

N.R. SMITH, et. al., "OECD/NEA Source Convergence Benchmark 1: Checkerboard Storage of Assembiles", Nuclear Energy Agency, http://www.nea.fr/html/science/wpncs/convergence /specifications/b1-checkerboard.pdf (2001).

D.B. MACMILLAN, "Monte Carlo Confidence Limits for IteratedSource Calculations", Nucl. Sci. Eng., 50, pp 73-87 (1973).

C.M. PERFETTI and B.T. REARDEN, "Continuous-Energy Eigenvalue Sensitivity Coefficient Calculations in TSUNAMI-3D", Proc. MEEC 2013, Sun Valley, ID, May 5-9 2013, on CD-ROM.

\section{Appendix A}

Throughout the analysis presented in Section II, it was assumed that the fission neutron emission spectrum $\chi(E)$ was independent of the incoming neutron energy. This is a usual assumption for the analysis of thermal reactors, but may introduce approximation for fast reactors. In practice, with MCNP6 and most other Monte Carlo codes, $\chi(E)$ is indeed a function of the energy of the incident neutron causing the fission. The analysis in Section II is unchanged, however, if $\chi(E)$ is replaced by $\chi(\vec{r}, E)$, defined as the average emission spectrum at position $\vec{r}$.

$\chi(\vec{r}, E)=\frac{\iint d E^{\prime} d \hat{\Omega}^{\prime} \chi\left(\vec{r}, E^{\prime} \rightarrow E\right) v \Sigma_{F}\left(\vec{r}, E^{\prime}\right) \Psi\left(\vec{r}, E^{\prime}, \hat{\Omega}^{\prime}\right)}{\iint d E^{\prime} d \hat{\Omega}^{\prime} v \Sigma_{F}\left(\vec{r}, E^{\prime}\right) \Psi\left(\vec{r}, E^{\prime}, \hat{\Omega}^{\prime}\right)}$

To use forward fission source modes calculated from the fission matrix in fixed source calculations, this energyaveraged spectrum would need to be tallied consistently with the tallies for the matrix elements during the course of the Monte Carlo simulation, but that is fairly straightforward. Regarding the analysis in Section II, the use of an average spectrum $\chi(E)$ introduces an additional approximation, in order to arrive at the equivalence statement of Eqs. (26). This concern is similar to the spatial weighting of the fission matrix elements. It is assumed that with a fine-enough mesh, this approximation would introduce a negligible degree of deviation from the aforementioned equivalence and the true forward fission source modes. The reasoning behind the assumption is similar to the discussion regarding Eqs. (26) and (27). 